\title{
1 Cellular senescence and quiescence are associated with altered ribosomal
}

\section{RNA methylation and processing}

3 Guohuan Yang ${ }^{1,2}$, Clemens Heisenberger², Isabelle C. Kos-Braun ${ }^{1}$, Norbert Polacek ${ }^{3}$, Johannes

4 Grillari², Markus Schosserer ${ }^{2, *}$ and Martin Koš ${ }^{1, *}$

5 1. Biochemistry Center (BZH), Heidelberg University, Heidelberg, Germany

6 2. Institute of Molecular Biotechnology, Department of Biotechnology, University of Natural Resources

7 and Life Sciences, Vienna, Vienna, Austria.

8 3. Department of Chemistry and Biochemistry, University of Bern, Bern, Switzerland

$9 *$ These authors contributed equally

Total: 10289 characters; 


\begin{abstract}
The 2'-O-methylation (2'-O-Me) of ribosomal RNA (rRNA) shows plasticity potentially associated with specific cell phenotypes. We used RiboMeth-seq profiling to reveal 2'-O-Me patterns specific to stress induced premature senescent (SIPS), quiescent and proliferating human dermal fibroblasts. The altered methylation levels in SIPS and quiescence partially correlated with the expression of specific snoRNAs but not fibrillarin. Senescence and quiescence were accompanied by a trend towards preferred usage of one of the two alternative ribosome biogenesis pathways.
\end{abstract}

\title{
Introduction
}

The accumulation of senescent cells is one of the main drivers of biological ageing mainly due to the characteristic pro-inflammatory senescence-associated secretory phenotype (SASP) (Coppe et al., 2010). Eliminating senescent cells extends the healthy lifespan of mice (Baker et al., 2016; Xu et al., 2018). Thus, strategies to deplete senescent cells or block the SASP offer therapeutic opportunities. Ribosomes represent a promising novel target, as delayed rRNA processing promotes induction of senescence by activation of p53 (Nishimura et al., 2015), and accumulation of pre-ribosomes maintains the senescent state by engaging retinoblastoma protein (Lessard et al., 2018). The protein composition of ribosomes in senescent cells was found to vary (Shi et al., 2017), but other ribosome features, such as rRNA modifications, were not yet explored.

The 2'-O-methylation (2'-O-Me), the most abundant modification in rRNA, is introduced by ribonucleoprotein complexes of four conserved proteins, including the methyltransferase fibrillarin (FBL), and a small nucleolar RNA (snoRNA) specifying the methylated site (Kiss, 2002). Recently, the plasticity of the 2'-O-Me modification of rRNA in response to stress was shown to contribute to the heterogeneity of ribosomes and affect their translational activity (Erales et al., 2017; Krogh et al., 2016). Here we show that the rRNA 2'O-methylation is altered in senescent and quiescent human cells. 


\section{Results and discussion}

We induced senescence in the human dermal fibroblasts (HDF) from three different donors by exposure to hydrogen peroxide (Lämmermann et al., 2018) and confirmed their senescent phenotype by changes in morphology, increased senescence-associated $\beta$ Galactosidase activity (SA- $\beta$-gal) and absence of proliferation by incorporation of 5-Bromo-2'deoxyuridine (BrdU) (Supp. Inf. Fig. S1). The contact-inhibited quiescent (Q) and proliferating (P) cells derived from each donor served as controls.

We used RiboMeth-seq to profile 104 2'-O-Me sites in the rRNA of SIPS, Q and P cells and assigned the RMS score $(0=$ non-methylated, $1=$ fully methylated $)$ to each modified position as described (Krogh et al., 2016) (Fig. 1 and Supp. Inf. Tables S2, S3). While the majority of sites were highly methylated (RMS > 0.9), the scores of nine positions in SIPS and Q cells appeared lower (SSU-C1391, LSU-C3680) or higher (SSU: C797, G867, C1272 and G1447; LSU: G1303, G3723 and G4588) compared to P cells (Fig. 1). The methylation at SSUC797, G867, C1272 and LSU-G1303 was nearly identical in Q and SIPS but different in P cells, pointing to a general role in proliferation. The SSU-C1391, G1447, and LSU- C3680, G3723 methylation in SIPS differed from P or Q cells suggesting a specific association with SIPS, however the biological variance between donors at these sites precludes a conclusion from the current data (Fig. 1; Supp. Inf. Tables S2, S3).

The RMS scores were overall slightly higher in $\mathrm{Q}$ and SIPS than $\mathrm{P}$ cells. In a recent study, the expression levels of FBL affected methylation at specific positions (Sharma et al., 2017). However, in our model the methylation in Q/SIPS and P cells did not correlate with FBL expression, which did neither change at mRNA nor at protein level in in any of the tested conditions (Supp. Inf. Fig. S1D-F).

In contrast, methylation at the hypomodified sites correlated with expression of snoRNAs guiding modification at those sites. Even small methylation changes were accompanied by comparable alterations in the snoRNAs levels (Fig. 1BD, Supp. Inf. Fig. S2, S3). Importantly, the expression of all snoRNAs for the hypomodified sites was very low and thus the hypomethylation might be due to the limiting levels of the snoRNAs. 

(Lessard et al., 2018; Nishimura et al., 2015), the 47 pre-rRNA did not accumulate in SIPS revealing that neither transcription nor early processing were affected (Fig. 2ABC). Conversely, the relative levels of $21 \mathrm{~S}$ and $18 \mathrm{~S}-\mathrm{E}$ pre-rRNAs decreased in SIPS, indicating an altered kinetics of the late SSU biogenesis (Fig. 2DE). Intriguingly, Q and SIPS cells showed a trend towards preference for the rRNA processing pathway B, represented by an increase in the $30 \mathrm{~S}: 41 \mathrm{~S}$ prerRNA ratio (41S and 30S pre-rRNAs are produced only by the pathway A or B respectively) (Fig. 2A and 2FGH). A similar switch between two pre-rRNA processing pathways in response to stress was described in yeast (Kos-Braun et al., 2017). Alternative pathways can provide means to produce distinct ribosomes.

In summary, we identified nine 2'-O-Me sites that appear to be differentially modified in non-proliferating cells (Q and SIPS). Their methylation correlated with the snoRNAs levels that are likely at limiting concentrations. Upon growth arrest, the pre-rRNA processing in SIPS and Q cells shifted towards the pathway B. Our results provide a basis for further study of ribosomes in cellular senescence as plausible targets for novel therapeutic interventions.

\section{ACKNOWLEDGEMENTS}

We thank Uschi Göbels and Elena Stelzer for technical assistance. GY was supported by the China Scholarship Council (201608310107). The work was supported by Deutsche Forschungsgemeinschaft 280594475 to MK, Austrian Science Fund (FWF) I2514 to JG and Herzfelder'sche Familienstiftung P30623-B26 to MS.

\section{AUTHOR CONTRIBUTIONS} experiments and analyzed data. GY, MS and MK wrote the paper. 
bioRxiv preprint doi: https://doi.org/10.1101/2020.04.01.019653; this version posted April 2, 2020. The copyright holder for this preprint

(which was not certified by peer review) is the author/funder, who has granted bioRxiv a license to display the preprint in perpetuity. It is made available under aCC-BY-NC 4.0 International license. 
Baker, D. J., Childs, B. G., Durik, M., Wijers, M. E., Sieben, C. J., Zhong, J., ... Deursen, J. M. Van. (2016). Naturally occurring p16 Ink4a -positive cells shorten healthy lifespan. Nature, 530(7589), 184-189. DOI:10.1038/nature16932

Coppe, J. P., Desprez, P. Y., Krtolica, A., \& Campisi, J. (2010). The senescence-associated secretory phenotype: The dark side of tumor suppression. Annu Rev Pathol, 5, 99-118. DOI:10.1146/annurev-pathol-121808-102144

Erales, J., Marchand, V., Panthu, B., Gillot, S., Belin, S., Ghayad, S. E., ... Diaz, J.-J. (2017). Evidence for rRNA 2'-O-methylation plasticity: Control of intrinsic translational capabilities of human ribosomes. Proceedings of the National Academy of Sciences, 114(49), 12934-12939. DOI:10.1073/pnas.1707674114

Kiss, T. (2002). Small nucleolar RNAs: An abundant group of noncoding RNAs with diverse cellular functions. Cell, 109(2), 145-148.

Kos-Braun, I. C., Jung, I., \& Koš, M. (2017). Tor1 and CK2 kinases control a switch between alternative ribosome biogenesis pathways in a growth-dependent manner. PLoS Biology, 15(3). DOI:10.1371/journal.pbio.2000245

Krogh, N., Birkedal, U., Christensen-Dalsgaard, M., Nielsen, H., Jansson, M. D., Häfner, S. J., ... Lund, 7884-7895. DOI:10.1093/nar/gkw482 Branco, A. D., ... Grillari, J. (2018). Blocking negative effects of senescence in human skin fibroblasts with a plant extract. Npj Aging and Mechanisms of Disease, 4(1). 
Lessard, F., Igelmann, S., Trahan, C., Huot, G., Saint-Germain, E., Mignacca, L., ... Ferbeyre, G. (2018). Senescence-associated ribosome biogenesis defects contributes to cell cycle arrest through the Rb pathway. Nature Cell Biology, 20(7), 789. DOI:10.1038/s41556-018-0127-y

Mullineux, S.-T., \& Lafontaine, D. L. J. (2012). Mapping the cleavage sites on mammalian pre-rRNAs:

Nishimura, K., Kumazawa, T., Kuroda, T., Katagiri, N., Tsuchiya, M., Goto, N., ... Kimura, K. (2015). p53 Activation. Cell Reports, 10(8), 1310-1323. DOI:10.1016/j.celrep.2015.01.055

Sharma, S., Marchand, V., Motorin, Y., \& Lafontaine, D. L. J. (2017). Identification of sites of 2'-O-

$$
\text { methylation vulnerability in human ribosomal RNAs by systematic mapping. Scientific }
$$

Shi, Z., Fujii, K., Kovary, K. M., Genuth, N. R., Röst, H. L., Teruel, M. N., \& Barna, M. (2017). Heterogeneous Ribosomes Preferentially Translate Distinct Subpools of mRNAs Genomewide. Molecular Cell, 67(1), 71-83.e7. DOI:10.1016/j.molcel.2017.05.021 
148 Fig. 1: RiboMeth-seq and snoRNA expression. (A, C) Average RMS scores for each 2'-O-methylated 149 nucleotide in 18S (A) and 28S (C) rRNAs from proliferating (P), quiescent (Q) and senescent (SIPS) 150 HDFs from three donors (HDF76, HDF161, HDF85). Error bars represent standard deviation. (B and

151 D) Relative levels of snoRNAs guiding modification at the variable sites. Values were corrected for 152 loading by snoRNA57 (northern) and 5.8S RNA (qRT-PCR).

154 Fig. 2 Pre-rRNA processing in SIPS. (A) Scheme of the human pre-rRNA processing (modified from 155 (Mullineux \& Lafontaine, 2012). (B) Northern blots of total RNA using two probes to detect different 156 pre-rRNAs shown on the right. (C-H) Quantification of changes in pre-rRNAs expressed as ratios: (C) 157 47S:28S; (D) 18S-E:47S; (E) 21S:47S; (F) 30S:41S; (G) 30S:47S; (H) 41S:47S. 


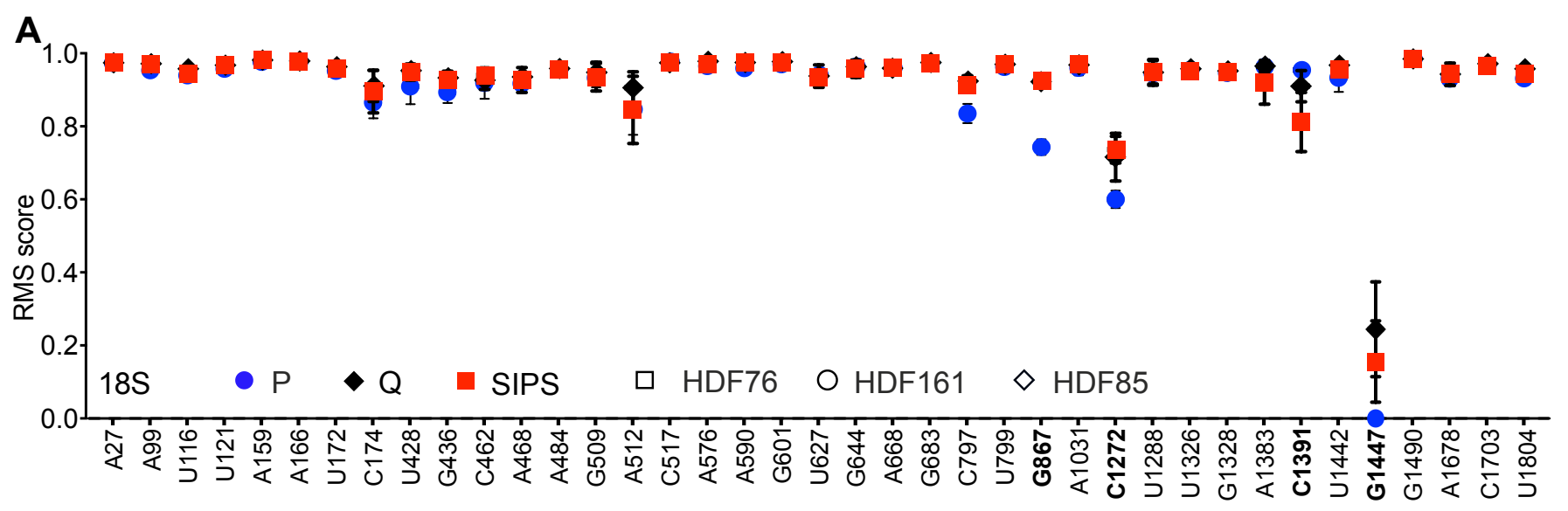

B RMS qRT-PCR

Northern

RMS

qRT-PCR

Northern
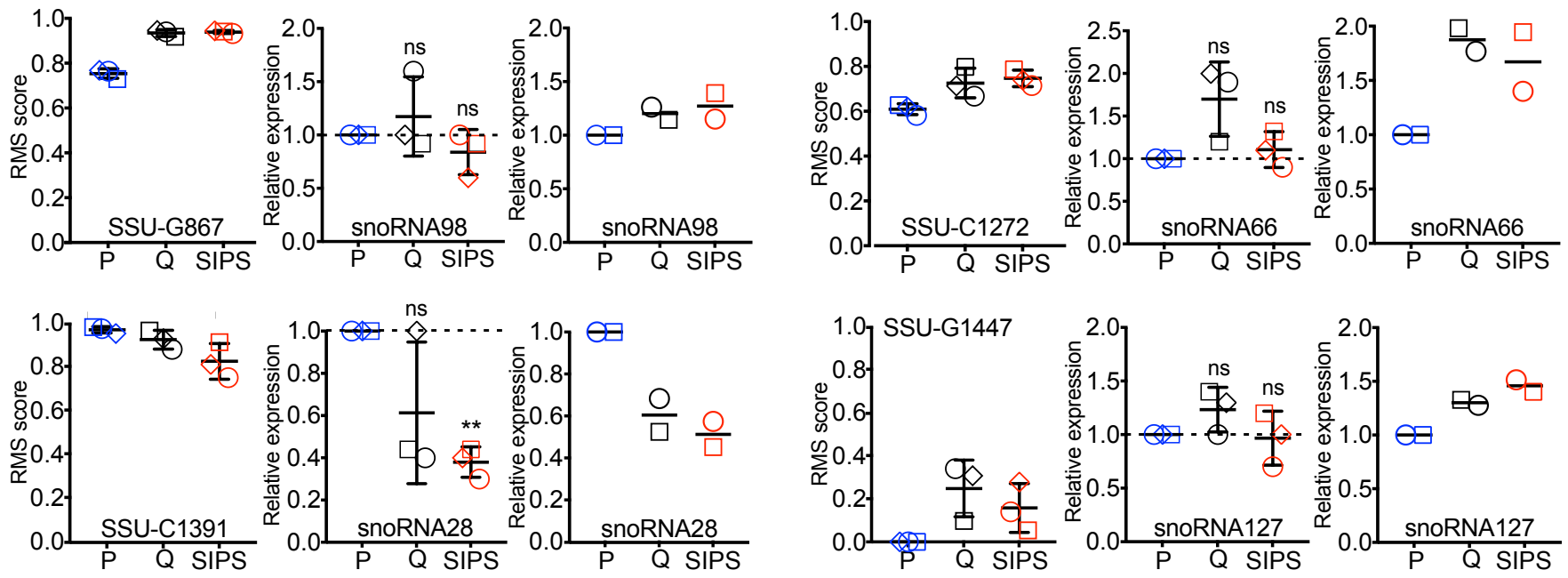

C

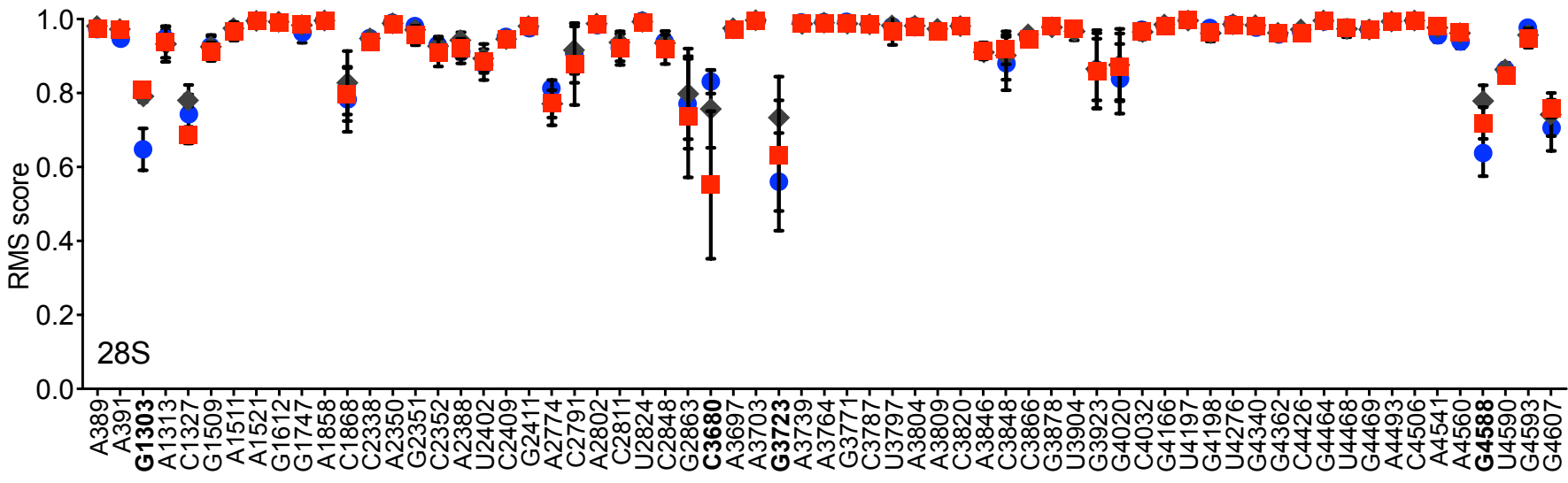

D

RMS

qRT-PCR

Northern

RMS

qRT-PCR

Northern

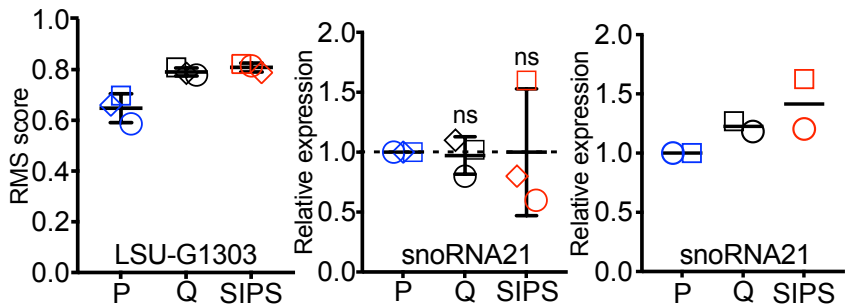

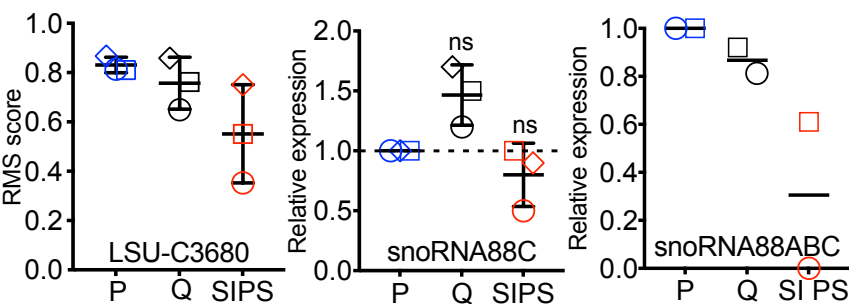

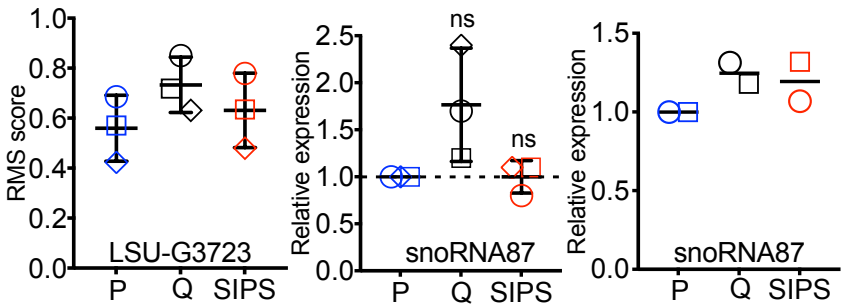

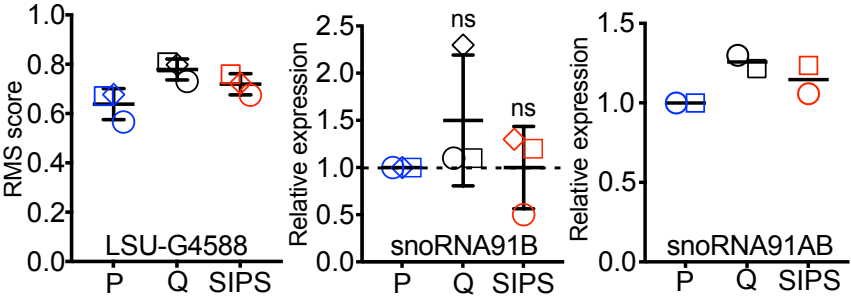


bioRxiv preprint doi: https://doi.org/10.1101/2020.04 01.019653: this version posted April 2.2020 . The copyriaht holder for this preprint (which was not certified by peer review) is the author/funder, who has granted bioRxiv a license to display the preprint in perpetuity. It is made available under aCC-BY-NC 4.0 International license.

A

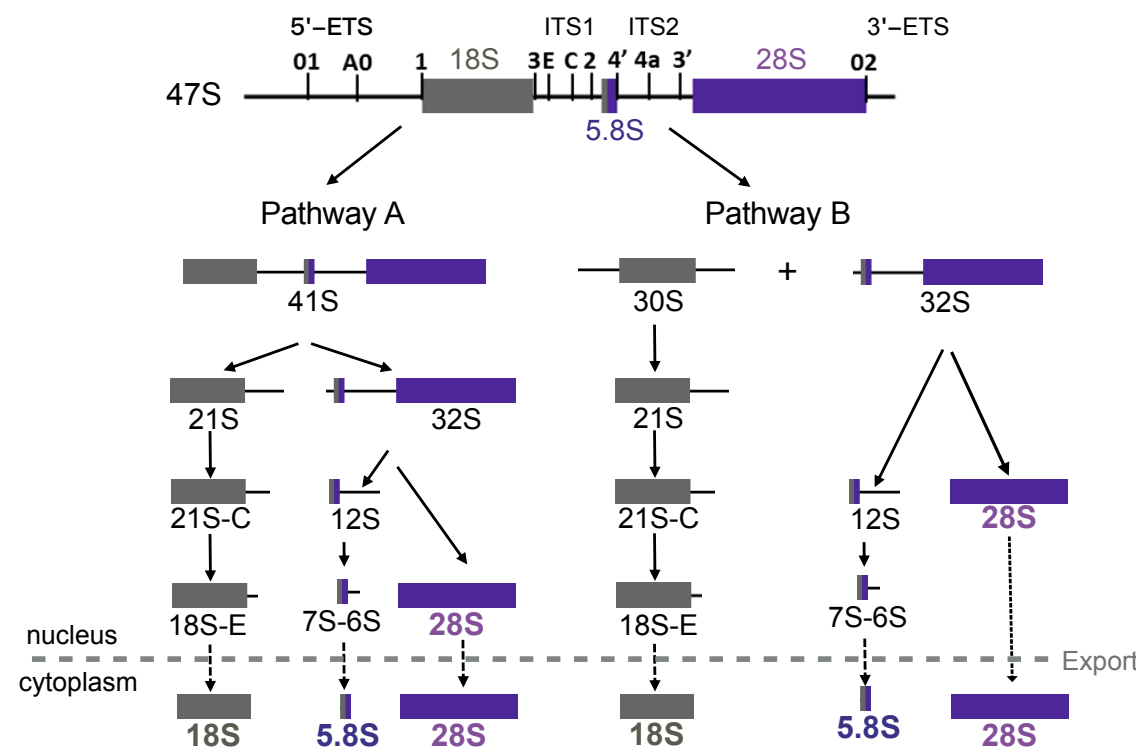

B
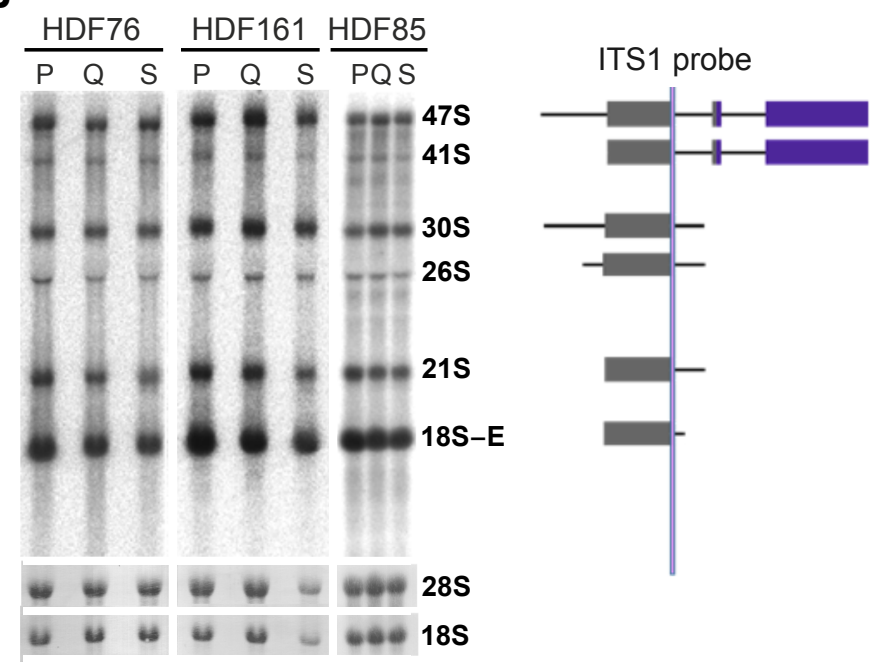

$\frac{\mathrm{HDF76}}{\mathrm{P} Q \mathrm{~S}} \frac{\mathrm{HDF} 161}{P \quad \mathrm{~S}} \frac{\mathrm{HDF85}}{\mathrm{PQS}}$

ITS2 probe
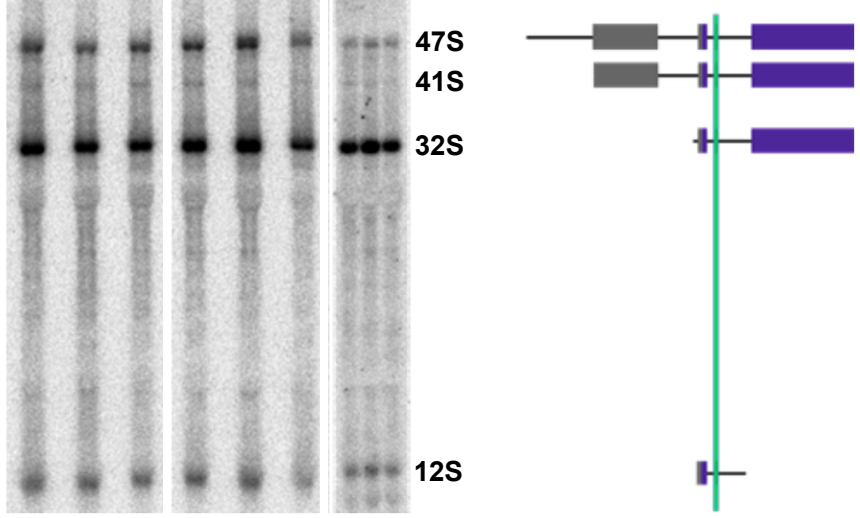

C

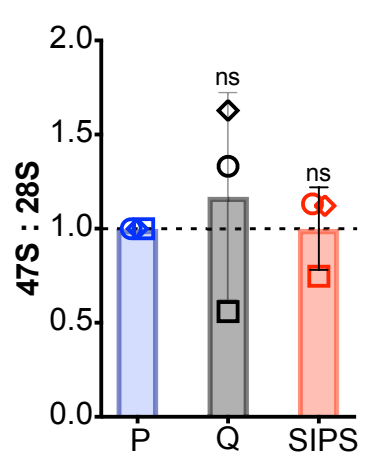

$\mathbf{F}$

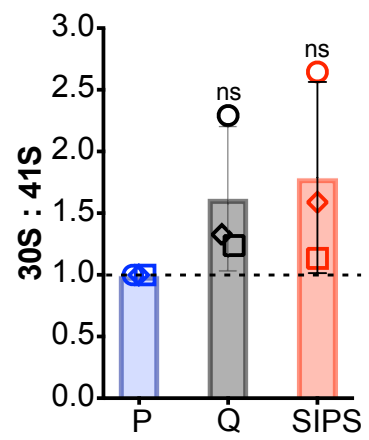

D

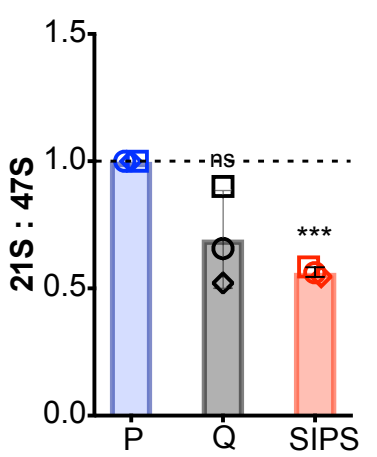

G

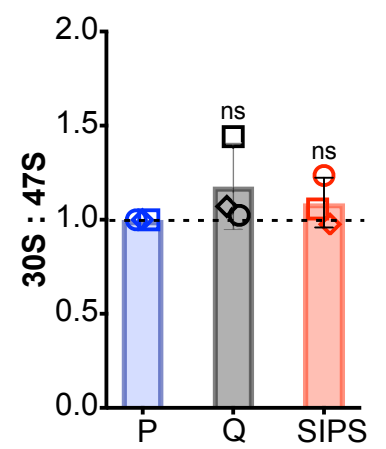

E

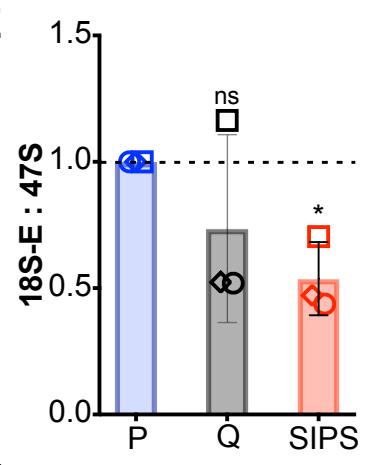

H

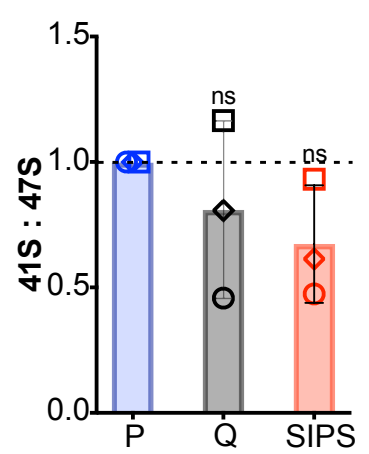

$\square$ HDF76

○ HDF161

$\diamond$ HDF85 